\title{
Developing National Functional Accreditation Model for Primary Healthcares with Emphasis on Family Practice in Iran
}

\author{
Jafar Sadegh Tabrizi', Farid Gharibi ${ }^{2 * *}$ \\ 'Tabriz Health Services Management Research Center, Tabriz University of Medical Sciences, Tabriz, Iran \\ ${ }^{2}$ Food Safety Research Center (Salt), Semnan University of Medical Sciences, Semnan, Iran
}

\begin{abstract}
Background: Accreditation is an approach toward quality improvement which has been increasingly implemented in healthcare. This study aimed at developing a national functional accreditation model for primary healthcare with emphasis on family practice in Iran.

Methods: This mixed-method study utilizes a set of research methods purposefully. Initially, the reference models were used for benchmarking accreditation standards through a systematic review. Then, the primary accreditation standards were developed and then they were assessed and approved by the experts of the field via Delphi technique. In the following and after developing essential parts of the standards, the necessary changes in developed model were done according to the pilot test results.

Results: The results of systematic review suggested the superiority of accreditation models of the United States, Australia, Canada, and the United Kingdom globally; and the models of Jordan, Saudi Arabia, Lebanon, and Egypt in Eastern-Mediterranean region. Then, the primary standards including 39 functional standards with 231 measures were developed according to the benchmarked models, and were approved by the experts in Delphi-based study. In pilot test step, the compliance rate of developed standards by primary healthcare centers was calculated $61.61 \%$ and $26.37 \%$ for self-evaluation and external evaluation phases, respectively.

Conclusion: Regarding the comprehensiveness of developed accreditation model due to its focus on all functional dimensions and the consensus over the developed standards by the experts, it can be an underlying ground for the establishment and evaluation of functional improvement programs in Iranian primary healthcare system.
\end{abstract}

Keywords: Primary Health Care; Family Practice; Accreditation

Received: February 14, 2020, Revised: April 14, 2020, Accepted: April 16, 2020

*Corresponding Author: Farid Gharibi https://orcid.org/0000-0001-9504-4660

Tel: +98-9181332935, Fax: +98-02334544842, E-mail: gharibihsa@gmail.com 


\section{INTRODUCTION}

Nowadays, accreditation has been increasingly utilized in health systems. More than 60 accrediting organizations are active worldwide, and international accreditation is developing surprisingly. ${ }^{1)}$ In its initiation, accreditation was implemented on hospitals and geriatric care centers and then it transferred to homecare services, hospice care, and finally it extended to the primary services. Accreditation is a process of self-assessment and external evaluation by peer experts on the basis of developed standards to achieve them and subsequently continuous quality improvement in health care organizations. It wants to achieve to a satisfactory functional level of input, processes and output for which health organizations are established. Of the positive impacts of health organizations' accreditation is the constant promotion of quality and safety, organizational culture, compatibility of healthcare with clinical guidelines, standardization of processes and establishment of functional management. It is obvious that functional improvement of healthcare organizations is achieved via affecting inner processes and culture, training, and putting emphasis on competitive merits. ${ }^{2)}$

Studies have shown that most of the health centers have considered accreditation as a beneficial investment for its significant effect in promoting safety and quality and take it as the first step to achieve promotion in healthcare services. Nevertheless, accreditation programs can promote the function of healthcare providing organization if they could provide a set of comprehensive and proportionate accrediting standards, in different functional aspects, for a successful accreditation. ${ }^{3)}$ It is clear that focus on one or more functional aspects, due to the ignorance or little emphasis on other important aspects, can hamper the organization's general function and thus, makes it necessary to define and evaluate the function of health organizations at different dimensions and then provide good balance between them. Therefore, accreditation standards must have enough comprehensiveness and reflect the aims and cover all key functions and processes of health centers. $^{4)}$

Primary healthcare systems in developing countries are facing numerous challenges including patients' dissatisfaction with violating their main rights, low quality of care, inefficient management in health centers, increasing costs due to the low efficiency of provided care, and so forth. The health system ranking which was done by the world health organization in annual health report 2000 revealed that the Iran's health system has not satisfactory status in functional domains such as accessibility, equity, equality, efficiency, and quality. Although several initiatives such as clinical governance, evidence-based medicine, and accreditation program were started in recent years to improve the health system, all of them are related to hospital care and there is huge neglecting from functional improvement in primary health care. Therefore, it is vitally important to promote the functional status of primary health care system by developing a proper accreditation programs with high quality functional standards. ${ }^{5,6)}$ Undoubtedly, developing and implementing a comprehensive, efficient and function-oriented accreditation program can improve organizational and clinical function in Iranian primary healthcare centers. ${ }^{6)}$ Given the absence of any efficient and function-oriented accreditation model in Iranian public health, the present study was conducted to develop a national functional accreditation model for primary healthcare with emphasis on family practice in Iran.

\section{METHODS}

This is a mixed-method study. First, the utilized approach to develop accreditation model was demonstrated according to scientific evidence. Next, the valid sources were identified by conducting a systematic review to develop accreditation standards. Then, primary accreditation standards were developed based on selected sources and they were approved by the experts through Delphi method. After that, the key components were developed for the developed standards. Finally, the necessary changes were made based on pilot test results (Figure 1).

There are many accreditation models worldwide which are valuable for benchmarking in developing new one; ${ }^{7)}$ therefore, they must be identified using literature review and relevant sources such as International Society for Quality in Healthcare (ISQua) website. ${ }^{8)}$ So the researchers conducted a systematic review to select the best accredita-

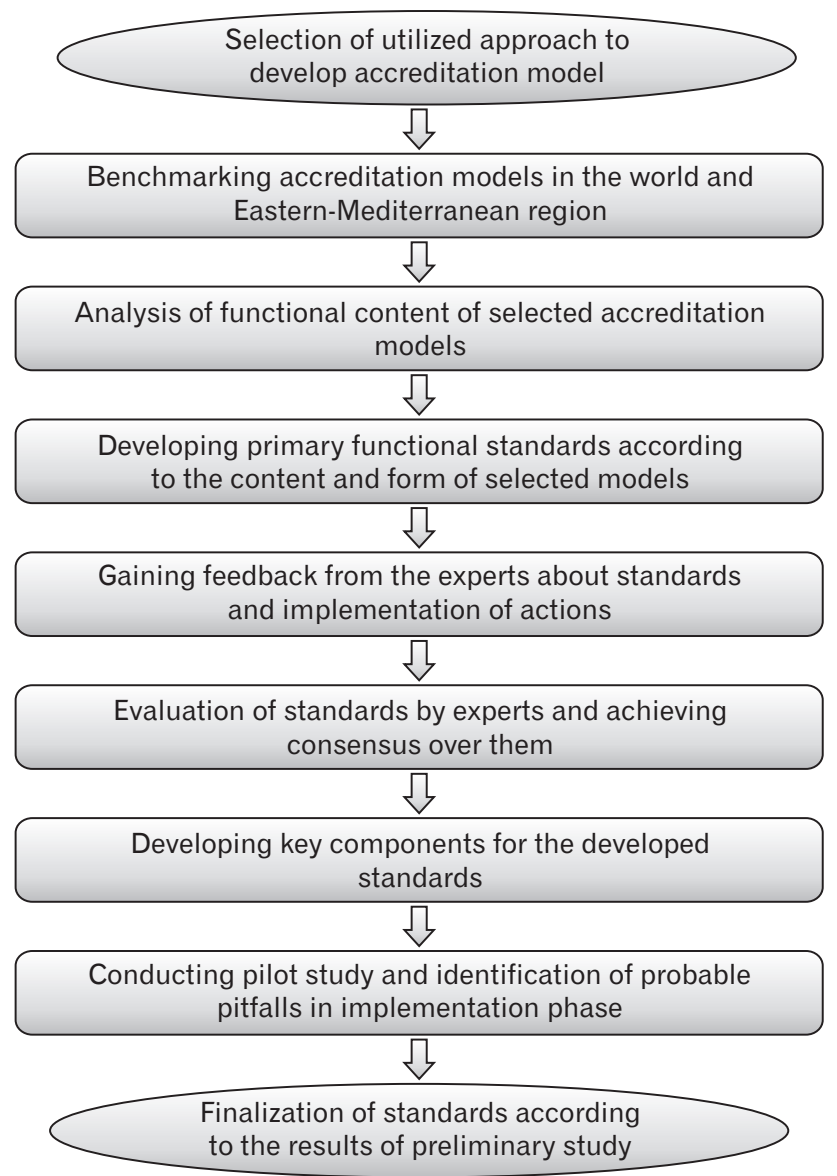

Figure 1. The process of developing functional accreditation model for primary healthcare in Iran. 
tion models in the field of primary healthcare in PubMed, Ovid, Medline, Scopus, Science Direct, SID (Scientific Information Database), and Magiran database/search engine. In this regard, a number of 9,051 articles were reviewed and the final conclusion was made about the referential models for benchmarking based on 19 included articles. ${ }^{7)}$ It must be noted that the main basis in the content analysis of benchmark accreditation models is to focus on the functional indexes in health system. Then, the content of benchmarked models was assessed for developing preliminary functional domains and standards. $^{9,10)}$

In continue, the primary developed accreditation standards were assessed by 23 experts via the Delphi technique based on "validity" and "feasibility" criteria in a 9-degree scale. ${ }^{11,12)}$ The experts were selected purposefully, it means that the people who were able to provide the best and the richest views and experiences to the researchers were investigated. So the experts were selected from academic members in healthcare management, social medicine, and family physician fields; the people who developed national hospital accreditation program; and the experienced managers in primary healthcare field from the ministry of health and deputy of health in medical sciences universities. ${ }^{12)}$ After collecting data and analysis, if the median score which obtained from expert opinion was between 1-4, the standard was excluded from the study and if it was between 4-7, it was entered into the next step and if acquired 7, the standard was directly included in the study. ${ }^{13)}$ Afterward, the necessary components including the content statement, measurable components, evaluation process, scoring pattern, and rating mechanism were developed to accreditation standards based on ISQua guidelines. ${ }^{12)}$

Given the gradual evolution of innovations and existence of tangible gap between their theoretical and implementation aspect initially, they must have pilot test study phase before final implementation so that the probable pitfalls would be identified and eliminated. ${ }^{4)}$ Thus, the Pilehroudi Health Center in Tabriz city, was selected as the pilot center and the self-assessment and external evaluation were done at it. Utilized tool in primary testing was a checklist that included developed standards and measures. Also, the primary standards were criticized and judged by managers and staffs of pilot health center in terms of the content, grouping method, writing, and evaluation process. Finally, their comments were included in the designed model.

Experts' freedom to participate or leave the study, official correspondences with all organizations and participating centers, taking consent from the participants, following privacy rules and respecting participants dignity, following anonymity rules and security of information, and using data exclusively in the present study were some ethical and moral consideration followed in the present study. The present investigation was also approved by the committee of ethics of Tabriz University of Medical Sciences.

\section{RESULTS}

\section{Selection of Used Approach to Develop Accreditation Model}

Standards are inseparable part of healthcare and accreditation models. They are "the ideal and achievable level of function that real function is judged upon them". ${ }^{14,15)}$ The main approaches in developing accreditation standards are benchmarking from other accreditation models, ${ }^{10)}$ accordingly the different countries' following from successful accreditation models and enjoying from their technical supports is the most common approach in this regard. For instance, accreditation models in Ireland, Lebanon, and South Korea have been adopted from Canada; Hong Kong from Australia; and Jordan and Egypt from the United States. ${ }^{6}$ In total, it can be claimed that the common trend in developing accreditation model is the content and form patterning of successful models in the world, utilizing experts' opinions, and conducting preliminary studies to adopt standards and measurements with endogenous requirements and necessities and utilizing the definitions provided by organizations such as ISQua., ${ }^{2,12)}$

\section{Benchmarking Well-Developed Accreditation Models}

The systematic review showed that, based on 11 criteria (including being the pioneer in the field of accreditation, effectiveness in developing novel accreditation models, focus on functional indexes of the health system, and having approval in different aspects by ISQua organization), the accreditation models in the United States, Canada, Australia, and the United Kingdom were the best models all around the world and accreditation models in Jordan, Egypt, Lebanon, and Saudi Arabia were the best models in Eastern-Mediterranean region. ${ }^{7)}$

\section{Developing Primary Functional Standards}

Functional domains which were extracted from benchmarked models were accessibility of care, community-oriented care, continuity of care, effective management, quality and safety of care, human resources management, information management, and patient's rights in delivered care. ${ }^{7)}$ By integrating the mentioned functional domains with valid evidence and gaining the opinions of managers and policy makers in Iran, it was tried to establish: functional domains of accessibility (geographical/physical accessibility, time accessibility, economic accessibility, and cultural accessibility), community-based services, continuity of care (information continuity, continuity of care providers, and continuity of different layers of health cares), quality and safety of care, evaluation of providing care, resources management, and research in health system. The developed functional model was actually established upon these 12 domains.

\section{Reaching Consensus over Primary Standards}

Delphi technique was applied to achieve consensus among experts on the measures and standards (with $87 \%$ response rate). Analysis of the Delphi technique results revealed a final model with 39 standards and 231 measures in functional domains. Functional domain of "accessi- 
bility of care" was the most extended domain with eight standards and 38 measures. Moreover, the functional domain of "planning in care delivery" was the most limited, as it included only two standards and nine measures (Table 1).

The highest score of "importance" for the presented measures in functional domains belonged to "human resource management" with the mean score of 8.30 and the lowest score received to "communityoriented care" with the mean score of 7.50. Moreover, the highest and lowest scores of "feasibility" with 7.40 and 6.81, respectively received to "human resource management" and "quality and safety of care" (Figures 2, 3).

\section{Developing Key Components for the Developed Standards}

The identified necessary parts for the developed accreditation model based on literature review and benchmarked models included content statement, measurable components, evaluation and measuring sys- tem, health centers rating system, and accreditation validation system. Content statement defined as "the statement explaining the philosophy behind the developing of a standard and its range covered". Thus, 39 content statements were developed based on benchmarked models and ISQua guidelines. ${ }^{10)}$ Measurable components are also one of the key parts of accreditation models which defined as "measures and components of accreditation models that contribute in objective assessment of standard". So, 231 measures were developed for the final 39 standards.

Literature review was revealed that 3-point Likert scale was proper for assessing compliance with accreditation standards, and this approach was selected. In this assessing approach, each choice was given the proportionate value (for example $1,0.5$, and 0 for full compliance, partial compliance, and incompliance with standards, respectively). In the following, the compliance rate of every measure, standard, accreditation domain, and all prerequisites of a model was calculated in the form of conformity percentage with standards. ${ }^{4,10)}$ The utilized system

Table 1. Results of Delphi method in the functional domains of final model

\begin{tabular}{|c|c|c|c|c|}
\hline Functional domain & $\begin{array}{l}\text { Total standards of the final } \\
\text { model }\end{array}$ & $\begin{array}{l}\text { Final approved } \\
\text { measures }\end{array}$ & $\begin{array}{l}\text { The mean score of measures } \\
\text { importance }\end{array}$ & $\begin{array}{c}\text { Mean of feasibility score of } \\
\text { measures }\end{array}$ \\
\hline Accessibility of care & 8 & 38 & 7.78 & 7.06 \\
\hline Community oriented care & 2 & 14 & 7.50 & 6.66 \\
\hline Continuity of care & 5 & 27 & 7.79 & 6.92 \\
\hline Quality and safety of care & 4 & 18 & 7.85 & 6.61 \\
\hline Patient's rights in delivered care & 3 & 28 & 8.08 & 7.04 \\
\hline Information management & 2 & 15 & 8.06 & 7.02 \\
\hline Human resource management & 2 & 14 & 8.30 & 7.40 \\
\hline Efficacy of care & 3 & 22 & 8 & 6.86 \\
\hline Planning in care delivery & 2 & 9 & 8.05 & 7.13 \\
\hline Evaluation of care & 2 & 10 & 8.17 & 7.03 \\
\hline Resource management & 4 & 24 & 8.10 & 7.02 \\
\hline Health system research & 2 & 12 & 7.66 & 6.79 \\
\hline
\end{tabular}

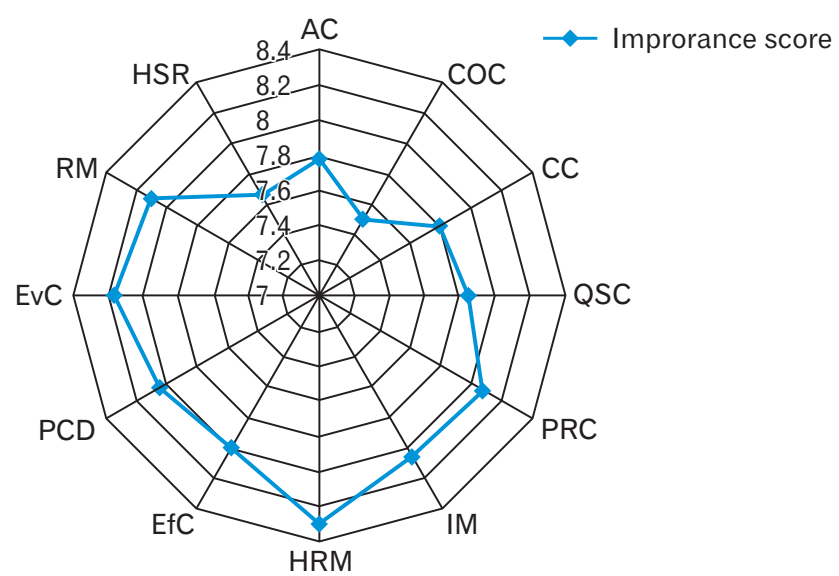

Figure 2. The mean scores of measures in "importance" domain. AC, accessibility of care; COC, community oriented care; CC, continuity of care; QSC, quality and safety of care; PRC, patient's rights in delivered care; IM, information management; HRM, human resource management; EfC, efficacy of care; $P C D$, planning in care delivery; $\mathrm{EvC}$, evaluation of care; RM, resource management; HSR, health system research.

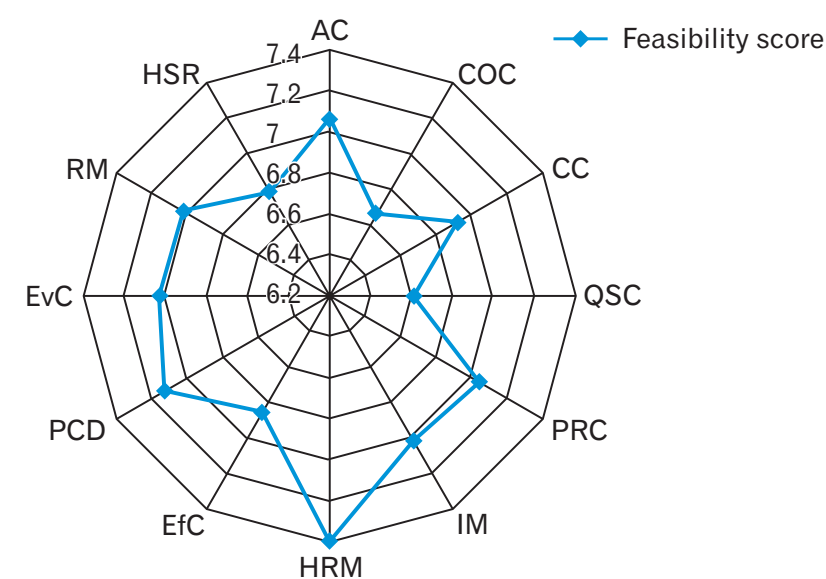

Figure 3. The mean scores of measures in "feasibility" domain. AC, accessibility of care; COC, community oriented care; CC, continuity of care; QSC, quality and safety of care; PRC, patient's rights in delivered care; IM, information management; HRM, human resource management; EfC, efficacy of care; PCD, planning in care delivery; $\mathrm{EvC}$, evaluation of care; RM, resource management; HSR, health system research. 
for rating process in the present investigation was suggested by ISQua in which the whole status of health organization is accredited based on four compliance levels of excellent, adequate, partially good, and weak compliance. It is obvious that, the logic behind the placement of an organization in a specific level is the main reason of the present condition and following that the necessary instructions are provided to the accredited organizations. ${ }^{4,10)}$

\section{Pilot Test and Finalization of Standards}

In this part, after providing managers of the studied health center with necessary training and counseling, the self-evaluation version of the accreditation was completed by the managers and experts. Then, the external evaluation was conducted by the evaluation team. In the following, the results obtained from self-evaluation phase and external evaluation were discussed and elaborated. Results showed that, in self-evaluation phase, the functional domains of "planning in care delivery" and "evaluation of care" with respectively $83.33 \%$ and $40 \%$ acquired the highest and lowest level of compliance of accreditation standards. In external evaluation, the functional domains of "resources management" and "quality and safety of care" respectively acquired the highest and lowest levels of compliance with $52.08 \%$ and $2.77 \%$. Furthermore, the total mean of compliance with the standards in both self-evaluation and external evaluation were respectively $61.61 \%$ and $26.37 \%$ (Table 2).

The designed standards were judged according to content, classification method, writing style, and so forth and were evaluated based on the perspectives of managers and personnel and their comments were considered in designing these standards. The necessity of integrating some measures with each other, necessity of separating some measures from each other and turning them into more than one measure, changing the themes of some developed standards to increase

Table 2. Compliance status of functional standards in the studied health center

\begin{tabular}{lcc}
\hline \multicolumn{1}{c}{ Evaluation domain } & Self-evaluation (\%) & External evaluation (\%) \\
\hline Accessibility & 52.26 & 38.63 \\
Geographical accessibility & 65 & 57.42 \\
Time accessibility & 40.9 & 18.18 \\
Economic accessibility & 62.5 & 37.5 \\
Cultural accessibility & 55.55 & 11.11 \\
Community oriented care & 78.57 & 10.71 \\
Continuity of care & 66.66 & 37.03 \\
Information continuity & 68.75 & 40.62 \\
Continuity of care provider & 63.63 & 31.81 \\
Quality and safety of care & 55.55 & 2.77 \\
Patient's rights in delivered care & 73.21 & 5.35 \\
Information management & 73.33 & 40 \\
Human resource management & 38.46 & 15.38 \\
Efficiency of care & 50 & 20.45 \\
Planning in care delivery & 83.33 & 22.22 \\
Evaluation of care & 40 & 25 \\
Resources management & 66.66 & 52.08 \\
Health system research & 58.33 & 8.33 \\
Total score & 61.61 & 26.37 \\
\hline
\end{tabular}

their compliance with the protocols and clinical guidelines, necessity of re-writing and correcting some measures, and finally the necessity of replacing some terms and words with more understandable and clear ones were among the comments that were implemented.

\section{DISCUSSION}

This study was conducted to develop national functional accreditation model about primary healthcare in Iran. The benchmarking process showed that accreditation models in the United States, Australia, Canada, and the United Kingdom were the best international models and models in Jordan, Saudi Arabia, Lebanon, and Egypt were the best in Eastern-Mediterranean region. In a study with the aim of identification of the best accreditation models in the field of hospital care it was revealed that the models in the United States, Canada, Australia, the United Kingdom, and New Zealand were the best international models and those of Egypt, Lebanon, and Jordan were the best and valid models in Eastern-Mediterranean region. ${ }^{6)}$ The results gained from these investigations showed that there was a close relation between their findings making it to claim that those countries that were successful in designing and implementing hospital care accreditation models have perceived the importance of moving toward developing primary health care accreditation models very earlier than other countries.

In the following, the functional domains were identified and their relevant standards were mixed with benchmarked models. These domains include: accessibility (geographical/physical, time, economic, and cultural accessibilities), community-oriented care, continuity of care (continuity of information, continuity of healthcare providers, continuity of care in different layers of care), quality and safety of care, patient's rights in delivered care, information management, human resources management, efficiency of care, planning in care delivery, evaluation of care, resources management, and health system research. They were demonstrated to be the 12 functional domains of developing accreditation models. In a study with the purpose of identification of functional domains in the accreditation of primary healthcare, continuity of care, accessibility, comprehensiveness of care, and coordination were confirmed as the core functional domains. ${ }^{16)}$ Another study identified accessibility, comprehensiveness of care, enjoyment, continuity of care, coordination, comprehensiveness of care, family-oriented care, community-oriented care, and cultural competence as the core functional domains. ${ }^{17)}$ In another investigation in Brazil, with the same objectives, accessibility, gate keeping, continuity of care, comprehensiveness of care, coordination, focus on family, community-oriented care, and focus on healthcare providers were the core functional domains in accreditation of primary health care. ${ }^{18)}$ In a similar study in Colombia, accessibility, gate keeping, continuity of care, comprehensiveness, coordination, focus on family, communityoriented care, professional training, and financial resources were the core functional domains. ${ }^{19)}$

Another interesting point in the findings of this section was that dif- 
ferent studies identified different domains and have put different levels of emphasis on these domains. Evaluation of these models revealed that each of the four benchmarked international models (the United States, Canada, Australia, and the United Kingdom) only emphasized on four or five functional models while the models of Eastern-Mediterranean region have almost covered all functional domains in their accreditation standards. The only exception was Lebanon that did not point out to three functional domains of accessibility, effective management, and patient's rights in delivered care. Moreover, none of the benchmarked models in developed countries focused on "departmental" standards and provided an exclusively functional accreditation model. While the benchmarked models of Eastern-Mediterranean region (except Lebanon) firmly put emphasis on the departmental standards. Given that the different accreditation models are designed and implemented to fulfill the needs of health centers on their way to promote the functions, one can conclude that the reason behind such differences is the purposefulness and requirement-based nature of accreditation standards. It is obvious that the accreditation models in developed countries only focused in limited number of functional domains for their better compliance with other functional domains in primary healthcare. It is while, the developing countries for their functional pitfalls in primary health care and inappropriate quality of delivered care in specialty filed needed to benefit from all functional and departmental accreditation domains.

The developed model in this study is more comprehensive than other accreditation models, in terms of covering functional domains. For instance, the American accreditation model only includes four functional domains of "community-oriented care," "effective management," "quality of care," and "safety of care" or the Canadian model only include five functional domains of "accessibility of care," "community-oriented care," "continuity of care," "safety of care," and "human resources management." The included measures also were not comparable to the present developed model. Of course, models in Jordan and Egypt pointed out all functional domains but the number of standards and measures for each domain was more limited in a way that for most domains only one standard and less than five measures were defined. Nevertheless, the present model has classified most of the domains and has implemented comprehensive measures and standards for the accurate evaluation and promotion of all domains.

The highest and lowest scores in the domain of "importance" in Delphi method were achieved by "human resources management" and "community-oriented care", respectively. The high score of human resources management highlights the high value of manpower in providing high quality community-based and preventive care, and its more tangibility in compared with others. ${ }^{20)}$ Also, the low score of community-oriented care in Delphi method can be due to centralized decision-making processes, dominance of individual-oriented care, and the absence of public participation in the field of primary health care in Iran. Numerous investigations have shown that Iranian health system, due to its centralization, low public participation in health-related activities, is in unsatisfactory condition in indexes such as justice, efficiency, responsiveness, and effectiveness. Therefore, it seems necessary to plan for the best intervention based on the regional priorities and increase the effect of health intervention and achieve justice, promote quality, increase financial resources. ${ }^{21)}$

The highest and lowest score of Delphi "feasibility" criteria for the developed measures was achieved in functional domains of "human resources management" and "quality and safety of care," respectively. The highest score in human resources management can be attributed to the tangibility of this domain when compared to the others and also due to high experience of experts in this field. In addition, the great bulk of healthcare in villages are provided by community workers who are well-trained and properly selected in terms of primary healthcare in Iran. ${ }^{22)}$ The lowest Delphi score of "quality and safety of care" domain in feasibility criteria can be attributed to the unfamiliar approaches of quality promotion in the field of primary health care in Iran resulted from absence of accreditation, clinical governance, clinical audit, and so forth in primary health care, when compared to the hospital care, limited and inconsistent administration of these programs, ${ }^{22)}$ complexity and difficulty of implementing quality promotion programs due to the lack of knowledge, low public participation, insufficient support from the authorities, financial deficits, lack of infrastructure, ${ }^{23)}$ insufficient devotion of the managers and physician in promoting the quality of their provided care, ${ }^{24)}$ and finally the large gap in this domain in achieving a desired level of qualitative function. ${ }^{25}$ )

Results of the pilot test of obtained model declared that in self-evaluation phase, the functional domains of "planning in care delivery" and "evaluation of care" acquired the highest and lowest compliance scores, respectively. The reason for highest score of planning can be due to clear perception and higher experience of assessed health managers and staffs from the planning processes due to existence serious resource limitation traditionally. The low score of evaluation of care is related to lack of accreditation system and professional evaluators in primary healthcare of Iran. Another reason is the complexity of evaluation process and insufficient knowledge and skill of managers and working staff in this regard. ${ }^{23,25,26)}$ However, acquiring the highest and lowest scores, respectively by planning and evaluation domains is a significant issue because these two domains are closely intermingled. The reason behind this close affinity is that the evaluation framework of an organization is done based on the strategy, objectives, and developed criteria which are determined in planning phase.

In external evaluation of the pilot test, the functional domains of "resources management" and "quality and safety of care" acquired the highest and lowest compliance to the standards of accreditation, respectively. The reason can be the daily attention of managers and staff to the health centers resources due to the tangibility of their management and the necessity of their constant and whole-dimensional supervision regarding the severe deficit in resources in the field of primary healthcare in developing countries. In contrary, quality is a novel issue in the field of primary healthcare and yet it has no place of regional planning of the country in macro micro levels. Problem detection and solving activities have not been carried out in the field of primary 
healthcare in a scientific and evidence-based manner and there is no accurate archiving process in this regard.

Moreover, the total mean compliance score for all standards in both self-evaluation and external evaluation phases were 61.61 and 26.73 , respectively, which connoted a huge gap between these phases. The main reasons behind such gap are wrong and non-evidence-based perception of managers and working staff of health centers about their own function, indifference of managers and working staff toward the purposive documentation of activities, obvious weakness in documentation of care, and notable pitfalls in necessary documents considered for the evaluation. It must be noted that the external evaluation only happens based on documents and evidence while, self-evaluation is mostly done on the perception of managers and working staff. It is almost clear that scientific and purposive documentation required for external evaluation and a proper self-evaluation accordingly (as a practice for external evaluation) contributes in more objectivity of evaluations and decreases the gap between scores obtained from selfevaluation and external evaluation. Furthermore, a quick glance at the results achieved by self-assessment and external evaluation suggests that the novel functional domains in accreditation model suffered from bigger gap between the scores achieved in self-evaluation and external evaluation phases. These novel domains include "community-oriented care," "quality and safety of care," "patient's rights in delivered care," and "health system research."

The strength points of this study are using evidence-based investigation through systematic review, purposive selection of experts from nationwide and their high response rate, participation of stakeholders in study phases, high obtained scores by the standards and their measures in Delphi technique phase, and implementing pilot test. Another advantage of the study is covering all 12 dimensions of primary health care in Iran by developing standards and measures for processes and relevant activities, focus on all core dimensions of a scientific evaluation (context, input, process, and results), and also emphasis on different levels of prevention in developed care standards. Of the limitations in the present study was the absence of English version of some models such as Indonesian accreditation program, different formats of benchmarked models (in terms of classification of standards and expression of functional domains), highly insufficient number of carried out studies in the field of accreditation in primary healthcare to draw comparison between them and our study, the potential challenges facing Delphi model (proper selection of participants in terms of specialty, to get insured of their anonymity, and proper management of abnormal and weird responses), ${ }^{11)}$ and also inveighing standards and measures.

Given the results, researchers have made executive suggestions to prevent from negative bureaucracy in accrediting organizations by arranging the field of information management and purposeful documentation, developing scientific and accurate accreditation processes, training professional evaluators with sufficient knowledge and skills for organizational evaluation and putting emphasis on their objective function, constant review of standards with the aim of promoting their capabilities specially in terms of flexibility and novelty, establishment of an independent accrediting organization to conduct effective accreditation, developing strategic and operational plan for the development and institutionalization of accreditation in primary healthcare in macro and micro levels, and also designing and implementing promotional interventions in the Pilehroudi Health Center to promote its current situation based on the obtained results from preliminary study.

In conclusion, the present study aimed at designing a comprehensive and evidence-based functional accreditation model to be implemented in the field of primary healthcare in Iran. It has been tried to use accurate and scientific tools and methods to achieve this aim. Different analyses have shown that despite great achievements in its initial decades of formation and also despite several merits such as adequate geographical accessibility due to extensive environmental infrastructure, primary healthcare in Iran still suffers from obvious pitfalls and challenges. Of the main weak points are functional problems (in terms of first contact point functions, continuity of care, comprehensiveness of care, and coordination), systemic problems (private and public healthcare providers, information system, payment system for providers), management problems (in five filed of monetary and facilities, human resources, planning and coordination, training and learning, and trusteeship), and also the challenges related to health system controlling factors (financial support, payments, organizing, legislation, and behavior). This system also faces the main challenges such as health transition, emerging health requirements, changing patterns of demand for primary care, and so forth. The success of this system in the promotion of health and public satisfaction depends on the elimination of these challenges in a scientific way. Given the opportunities to promote primary healthcare services in Iran and given that most of the above mentioned challenges are included in the present developed accreditation model, we hope that the utilization of the present model would result in significant efficiency in the elimination of facing challenges. The present model can be considered as a basis for longterm design and planning to promote the quality of primary healthcare services in Iran as the modifications in accreditation approaches can leave deep impact on care delivery and consequently on constant promotion of the quality of health care services. Due to the utilization of evidence-based and scientific methodologies in its implementation, the present study can be useful for those countries who want to promote and enhance the quality of their primary healthcare.

\section{CONFLICT OF INTEREST}

No potential conflict of interest relevant to this article was reported.

\section{ACKNOWLEDGEMENTS}

The authors would like to appreciate all the experts who wholeheartedly cooperated in the present study. We also thank all the managers and working personnel of the health centers for their participation in 
the pilot model and also the Tabriz Health Services Management Research Center for its financial support.

\section{ORCID}

Jafar Sadegh Tabrizi: https://orcid.org/0000-0002-1458-8672

Farid Gharibi: https://orcid.org/0000-0001-9504-4660

\section{REFERENCES}

1. Greenfield D, Braithwaite J. Developing the evidence base for accreditation of healthcare organisations: a call for transparency and innovation. Qual Saf Health Care 2009;18:162-3.

2. Shaw CD. Toolkit for accreditation programs. Melbourne: The International Society for Quality in Health Care; 2004.

3. Saleh SS, Bou Sleiman J, Dagher D, Sbeit H, Natafgi N. Accreditation of hospitals in Lebanon: is it a worthy investment? Int J Qual Health Care 2013;25:284-90.

4. Fortune T, O'Connor E, Donaldson B. Guidance on designing healthcare external evaluation programmes including accreditation. Dublin: International Society for Quality in Healthcare; 2015.

5. World Health Organization. Quality and accreditation in health care services: a global review. Geneva: World Health Organization; 2003.

6. Tabrizi J, Gharibi F. Systematic survey of accreditation models for designing a national model. Sci J Kurdistan Univ Med Sci 2011;16:95-109.

7. Tabrizi JS, Gharibi F. Primary healthcare accreditation standards: a systematic review. Int J Health Care Qual Assur 2019;32:310-20.

8. Gharibi F, Tabrizi JS. Development of an accreditation model for health education and promotion programs in the Iranian primary healthcare system: a Delphi study. Health Promot Perspect 2018;8: 155-62.

9. Jaafaripooyan E, Agrizzi D, Akbari-Haghighi F. Healthcare accreditation systems: further perspectives on performance measures. Int J Qual Health Care 2011;23:645-56.

10. International Society for Quality in Health Care. Guidelines and principles for the development of health and social care standards. 4th ed. Dublin: International Society for Quality in Health Care; 2015.

11. Kim YS, Han SH, Hwang JH, Park JM, Lee J, Choi J, et al. Development of the Korean framework for senior-friendly hospitals: a Delphi study. BMC Health Serv Res 2017;17:528.

12. Tabrizi JS, Gharibi F, Pirahary S. Developing of national accreditation model for rural health centers in Iran health system. Iran J Public Health 2013;42:1438-45.

13. Culley JM. Use of a computer-mediated Delphi process to validate a mass casualty conceptual model. Comput Inform Nurs 2011;29:272-9.

14. Greenfield D, Pawsey M, Hinchcliff R, Moldovan M, Braithwaite J. The standard of healthcare accreditation standards: a review of empirical research underpinning their development and impact. BMC Health Serv Res 2012;12:329.

15. Greenfield D, Civil M, Donnison A, Hogden A, Hinchcliff R, Westbrook J, et al. A mechanism for revising accreditation standards: a study of the process, resources required and evaluation outcomes. BMC Health Serv Res 2014;14:571.

16. Cassady CE, Starfield B, Hurtado MP, Berk RA, Nanda JP, Friedenberg LA. Measuring consumer experiences with primary care. Pediatrics 2000;105:998-1003.

17. Shi L, Starfield B, Xu J, Politzer R, Regan J. Primary care quality: community health center and health maintenance organization. South Med J 2003;96:787-95.

18. Macinko J, Almeida C, de Sa PK. A rapid assessment methodology for the evaluation of primary care organization and performance in Brazil. Health Policy Plan 2007;22:167-77.

19. Mosquera PA, Hernandez J, Vega R, Martinez J, San Sebastian M. Performance evaluation of the essential dimensions of the primary health care services in six localities of Bogota-Colombia: a cross-sectional study. BMC Health Serv Res 2013;13:315.

20. Kumar P, Khan AM. Human resource management in primary health care system. Health Popul 2013;36:66-76.

21. Dejman M, Falahat K, Baradaran Eftekhari M, Mahmoodi Z, Forouzan AS, Jorjoran shushtari Z, et al. Addressing social determinant of health in Iranian healthcare network: a proposed model. Hakim J 2018;18:1425.

22. Abbaszadeh A, Eskandari M, Borhani F. Changing the care process: a new concept in Iranian rural health care. Asian Nurs Res (Korean Soc Nurs Sci) 2013;7:38-43.

23. Farahbakhsh M, Nikniaz AR, Zakeri A. Assessment of health data management in primary health care of East Azerbaijan. J Health Syst 2010;2:51-8.

24. Gharibi F, Tabrizi JS. Challenges of the new Iranian accreditation system based on the requirements of the international society for quality in healthcare (ISQua). J Clin Res Gov 2015;17;4:1-6.

25. Ghaffari Sardasht F, Jafarnejad F, Jahani N. Applying Donabedian quality-of-care framework in assessing the structure of preconception care in urban health centers, Mashhad, 2012. J Mazandaran Univ Med Sci 2014;24:149-60.

26. Asadi F, Hosseini A, Moghaddasi H, Haydarabadi NN. Primary health care information systems in health centers of Tehran, Iran. Health Inf Manag 2012;9:1-10. 